\title{
„Warum heilen
}

\section{Wunden bei gestressten Patienten schlechter?“}

\author{
Frage an Frans Manch kniffelige physiologische Frage stellt sich \\ einem im physiotherapeutischen Berufsalltag. Solchen Fragen geht \\ Medizinjournalistin Mona Herz für physiopraxis auf den Grund und \\ stellt sie unserem Experten Frans van den Berg.
}

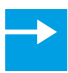

Dass Kortisol die Wundheilung beeinflusst, lässt sich in der täglichen Praxis häufig beobachten. Bei gestressten Patienten heilen Wunden oft deutlich schlechter als bei entspannten. Auch die Wissenschaft kommt zu diesem Ergebnis. Forscher untersuchten in verschiedenen Studien an Mensch und Tier, wie sich Stress auf die Wundheilung auswirkt. Sie fanden zum Beispiel heraus, dass sich das Infektionsrisiko nach Verletzungen bei gestressten Mäusen verglichen mit entspannten Artgenossen um das Dreifache erhöht [1]. Menschliche Wunden heilen bei Stress um 40 Prozent schlechter. Das zeigt eine Untersuchung an Studierenden, deren Wunden in den Semesterferien schneller verheilten als in der stressigen Prüfungszeit [2]. Zudem gibt es Hinweise, dass Verletzungen von Menschen, die in einer Beziehung mit generell aggressivem

Umgangston leben, langsamer abklingen als bei Menschen in harmonischen Beziehungen. Bei ihnen kann jedoch bereits ein einzelner Streit mit dem Partner dieselbe Wirkung hervorrufen [3]. Chronischer Stress, beispielsweise die Pflege eines Angehörigen oder anhaltende finanzielle Sorgen, kann die Wundheilung also

9

Die Wunden von Studierenden heilten in den Semesterferien um 40 Prozent schneller als in der Prüfungszeit. ebenso verlangsamen wie ein akuter Stressfaktor.

Eingeschränkte Entzündungsreaktion verlangsamt die Heilung $\rightarrow$ Warum Stress, beispielsweise in Form psychosozialer Belastungen, eine solch zentrale Rolle spielt, zeigt die Physiologie. Die Wundheilung zeichnet sich durch zwei wichtige Phasen aus: die Gewebeabräumung und den Gewebeaufbau. Ist die Blutung gestillt, beginnt innerhalb der Entzündungsphase die Gewebeabräumung. Hierbei „fressen“ Phagozyten, zum Beispiel 
Makrophagen, Unbrauchbares wie abgestorbenes Gewebe oder Schädliches wie Bakterien. Als Nächstes entsteht durch die Zytokinausschüttung eine Entzündungsreaktion, welche für den Heilungsprozess sehr wichtig ist. Sie stabilisiert die Wunde und schützt sie unter anderem vor schädlichen Mikroorganismen. Dabei kommt es zu den klassischen Symptomen: Erwärmung (Calor), Rötung (Rubor), Schwellung (Tumor) und Schmerz (Dolor). Die Erwärmung und Rötung entstehen durch die gesteigerte Durchblutung im Wundbereich, ausgelöst durch gefäßerweiternde Substanzen der Granulozyten und Makrophagen.

Durch die erweiterten Blutgefäße verringert sich die Geschwindigkeit des Blutflusses und die Gefäßpermeabilität erhöht sich. Das vereinfacht den Leukozyten die Arbeit, da sie sich leichter durch die Kapillarwand bewegen können. Gleichzeitig schwillt das Gewebe an, weil mehr Flüssigkeit und Plasmaproteine aus den Blutgefäßen in das Gewebe wandern. Für die erhöhte Durchlässigkeit der Gefäße sind beispielsweise Histamin oder Prostaglandin $\mathrm{E}_{2}$ verantwortlich. Diese Substanzen lösen zum Teil auch die lokalen Schmerzen aus.

Ist die Gewebeabräumung abgeschlossen, beginnt der Gewebeaufbau (Proliferationsphase), die tatsächliche Wundheilung. Dafür müssen sich Blutgefäße bilden (Angiogenese), und durch Bindegewebszellen (Fibroblasten) entsteht Kollagen. Eine Vielzahl von Signalstoffen bewirkt diese Prozesse, zum Beispiel ein lokal freigesetzter Wachstumsfaktor (basic fibroblast growth factor), der die Kollagenbildung anregt. Dieser ist essenziell für den Gewebeaufbau und den schnellen Wundverschluss. So ließ sich bei der Untersuchung mit den Studierenden in der Wundflüssigkeit der prüfungsgestressten Gruppe weniger von diesem Wachstumsfaktor nachweisen [2]. Zudem konnten andere Autoren mit dem gleichen Experiment zeigen, dass in den Granulozyten weniger Proteinbiosynthese stattfindet [4]. Die Forscher machen eine reduzierte Zytokinkonzentration an der Wunde und den damit eingeschränkten Entzündungsprozess für die verlangsamte Heilung verantwortlich.

Und hier kommt vermutlich das Kortisol ins Spiel: Bei Stress erhöht sich der Kortisolspiegel, wodurch wiederum weniger entzündungsfördernde (proinflammatorische) Immunstoffe ausgeschüttet werden. Kortison kommt daher in der Medizin als Entzündungshemmer zum Einsatz [5].

Hyperaktive Hypothalamus-Hypophysen-Nebennierenachse steigert Kortisolausschüttung $\rightarrow$ Bei einer Stressreaktion ist die Aktivität der Hypothalamus-Hypophysen-Nebennierenachse (HHNA) bedeutend. Diese ist bei gestressten Menschen meist hyperaktiv, was sich in einer gesteigerten Produktion verschiedener Stresshormone zeigt. Die höhere Konzentration dieser Hormone versetzt den Körper in einen „Stresszustand“. Beispielsweise sorgen das CRH (Corticotropin-Releasing-Hormon) und das ACTH (adrenocorticotropes Hormon) für die Ausschüttung von Adrenalin, Noradrenalin und Kortisol und sind so an einem höheren Blutdruck sowie einer gesteigerten Herzfrequenz beteiligt. Es ist allerdings zu beachten, dass jeder Mensch individuell auf Stress reagiert und hierbei Umweltfaktoren, soziales Umfeld, genetische Aspekte und die Funktionalität der endokrinen Systeme wichtige Rollen spielen.

Kortisol und wenig Sauerstoff behindern die Wundheilung $\rightarrow$ Dass Kortisol an der verzögerten Wundheilung beteiligt sein könnte, hat Padgett 1998 mit einem Experiment mit Mäusen untermauert: Chronisch gestresste Tiere bekamen einen GlukokortikoidAntagonisten verabreicht. Dieser hemmt die Wirkung von Glukokortikoiden, zu denen unter anderem auch Kortisol zählt. Obwohl die Tiere unter Stress standen, verheilten ihre Wunden vergleichbar schnell wie die der entspannten Mäuse [6]. Ebenso konnten Wissenschaftler um Gajendrareddy 2005 an Mäusen zeigen, dass Adrenalin die Hautgefäße verengt. Dadurch gelangt weniger Sauerstoff an die Wunde und die Heilung verzögert sich. Erhielten die gestressten Mäuse im frühen Stadium der Wundheilung zweimal täglich eine spezielle Sauerstofftherapie, heilten ihre Verletzungen wie die der entspannten Vergleichsgruppe [7].

Zwar werden in der täglichen Praxis Glukokortikoid-Antagonisten und Sauerstofftherapie wohl kaum zum Einsatz kommen, um die Wundheilung von gestressten Menschen zu verbessern. Hilfreich könnte es aber sein, wenn Physiotherapeuten ihre Patienten über den Zusammenhang von Stress und verzögerter Wundheilung informieren und mit ihnen stressabbauende Aktivitäten erarbeiten. Langfristig mündet beides im besten Fall auch noch in einen entspannten Alltag.

Mona Herz

Literaturverzeichnis www.thieme-connect.de/products/physiopraxis > „Ausgabe 11-12/16“

$\Rightarrow$ Frans beantwortet auch Ihre Frage!

Mit physiologischen Themen kennt sich Frans van den Berg bestens aus. Sie haben eine Frage, die er beantworten soll? Dann schreiben Sie sie uns: physiopraxis@thieme.de.

\section{谓 Gewinnen}

\section{Physiotherapie für alle Körpersysteme}

Wir verlosen ein Exemplar des Buches „Physiotherapie für alle Körpersysteme“ von Frans van den Berg. Wer gewinnen will, klickt bis zum 31. Dezember 2016 unter www.thieme.de/physiopraxis > „Gewinnspiel“ auf das Stichwort „Körpersysteme“. 